\title{
DIRECT ELECTRICAL MEASUREMENT OF THE PERMANENT POLARIZATION OF A FERROELECTRIC CHIRAL SMECTIC C LIQUID CRYSTAL $\left(^{*}\right)$
}

\author{
Ph. MARTINOT-LAGARDE \\ Université de Paris-Sud, Physique des Solides (**) 91405 Orsay, France
}

(Reçu le 19 août 1976, accepté le 18 octobre 1976)

\begin{abstract}
Résumé. - On oriente un échantillon de p-Hexyl-Oxy-Benzylidène-p'-Amino-2-Chloro-PropyleCinnamate, cristal liquide chiral ferroélectrique, dans sa phase smectique $C$, par un champ électrique alternativement égal à $+E$ et $-E$; sa polarisation permanente s'oriente de $+P$ à $-P$ en un temps ajustable avec l'amplitude de $E$. Ce temps est choisi pour que le courant électrique transitoire induit par cette variation de la polarisation soit discernable des courants de charge et de conduction de l'échantillon. L'intégrale de ce courant transitoire donne la valeur de $P$. On trouve $P=5 \times 10^{-2}$ Debye par molécule et par radian d'angle d'inclinaison. Cette méthode donne la première mesure directe de $P$ et sa variation avec la température.
\end{abstract}

Abstract. - A sample of p-Hexyl-Oxy-Benzylidne-p'-Amino-2-Chloro-Propyle-Cinnamate, Chiral liquid crystal, is oriented in the ferroelectric smectic C-phase by a square wave field $\pm E$; its permanent polarization orients itself from $+P$ to $-P$ in a time adjustable with $E$. This time is chosen to obtain an induced transient current, due to the polarization variation, distinguishable from the charge and conduction currents in the sample. The integral of this bump of current gives the value of $P: P=5 \times 10^{-2}$ Debye per molecule and radian of the molecular tilt angle. This method gives the first direct measurement of $P$ and its variation versus the temperature.

As shown by R. B. Meyer et al. [1], liquid crystals can be ferroelectric in the smectic C-phase if they are made of chiral molecules possessing a transverse permanent dipole. The measurement of that ferroelectrical polarization is important both for theoretical purpose and for possible applications. Up to now, two experiments have been designed to measure that polarization ; they use an electroelastic effect $[2,3]$ or a shear induced polarization [4]. Unfortunately they both give only the ratio of the polarization to an unknown twist elastic constant. More recently, a pyroelectrical experiment [5] has been reported which gives an estimation of the ferroelectrical polarization. However this experiment is unable to detect any temperature dependence of the spontaneous polarization, and the experimental effect reported seems to be opposite in sign to the theoretical results predicted by the authors. So this result seems to us very questionable.

In this paper we report a direct absolute electric measurement of the permanent polarization of the HOBACPC (p-Hexyl-Oxy-Bensylidne-p'-Amino-2-

(*) This work was supported by the French D.R.M.E., Contract $74 / 547$.

${ }^{(* *)}$ Associated with C.N.R.S.
Chloro-Propyle-Cinnamate). This compound was chosen because its permanent polarization, estimated by indirect measurement [3], is about ten times larger than the polarizations of the other known chiral $\mathbf{C}$ compounds. Dynamical analysis allows us to distinguish between the signal and conduction or capacity effects; this method allows also the analysis of temperature dependence of the polarization.

The principle of the experiment is the following : at a given temperature, the ferroelectric sample is oriented in one direction by a strong DC electric field $E$. This field is rapidly reversed; the dipoles turn themselves up in the opposite direction; we measure the current $I$ during this halfturn of the ferroelectric polarization. The integral of this current gives obviously the charge which has flowed through the sample. The voltage independent part of this charge corresponds to the charge due to the spontaneous polarization.

This experiment presents two difficulties : first it is necessary to reverse the ferroelectric dipoles by keeping an uniform texture; secondly we have to reverse the dipoles rapidly enough to induce a current, observable compared to the large conduction or capacitive current in the sample. 
The first point can be checked with optical methods. For the second point, calling $\tau$ the dipole reversing time, the current induced by the inversion of the macroscopic polarization $P$ is $I_{P} \sim 2 P S / \tau$ (where $S$ is the sample area). An estimate of $\tau$ is the relaxation time of the mean molecular rotation on the cone inside the smectic layers. For most ferroelectric compounds, the relaxation frequency has been measured [2] by a dielectric technique (about $400 \mathrm{~Hz}$ ) which means $\tau$ of the order of a few milliseconds. The optimum orienting field is then a square wave $\pm E$, symmetrical compared to ground, of a few milliseconds duration. In this case, just after the inversion of the field $E$, the total current flowing through the electrodes circuit which apply $E$ is expected to be the superposition of the trivial constant conduction term, of an exponential capacitive contribution, and of this transient current induced by the inversion of the ferroelectrical polarization.

In practice the sample is oriented in the A-phase between two obliquely evaporated $\mathrm{SiO}$ transparent electrodes giving a so called planar orientation; the sizes of the electrodes are $7 \mathrm{~mm}$ by $3.5 \mathrm{~mm}$. The thickness of the sample is $0.2 \mathrm{~mm}$ to avoid too large an influence of the electrodes anchoring and to allow a good orientation. The sample temperature is stabilized by an electric hotstage at better than $0.1^{\circ} \mathrm{C}$. Cooling the sample in the $\mathrm{C}$-phase induces the fingerprint texture, i.e. the helical texture with its axis parallel to the electrodes.

We apply the field with a square wave generator of $10 \mu$ s rise time giving a maximum field of $\pm 725 \mathrm{~V} / \mathrm{cm}$. When this maximum field is applied, the fingerprint texture disappears after one or two seconds if the frequency of the wave is lower than $700 \mathrm{~Hz}$. At low frequency $(1 \mathrm{~Hz})$ we see only a texture aligned in the plane of the electrodes and making with the normal of the smectic layers the tilt angle $\theta$ or $-\theta$ according to the sign of the applied field. This observation suggests that when the field is reversed, the motion of the molecules from $\theta$ to $-\theta$ is a half-circle about the normal to the layers. This motion is effected simultaneously by all the molecules in the sample and happens very quickly. There is no indication of any rewinding in the helical texture.

We measure the transient current $I$ by measuring the voltage drop across a serie resistor of $1 \mathrm{M} \Omega$; smaller than the sample resistance (30 M $\Omega$ ). This voltage drop is observed on an oscilloscope triggered by the square wave generator. The sweep time of the oscilloscope is $0.5 \mathrm{~ms}$ per division and the sensitivity $0.5 \mu \mathrm{A}$ per division. Figure 1 shows the time recording of $I$ on the screen. $I=0$ is indicated by the horizontal line. Twenty sweeps are superimposed in this image, ten when the sample is at a fixed temperature in the $\mathrm{A}$ phase and ten in the $\mathrm{C}$ phase. This gives an estimate of the dispersion of the current and allows the comparison of the signal in the $A$ and in the $C$ phases. In the A phase for $E=725 \mathrm{~V} / \mathrm{cm}$, we see only

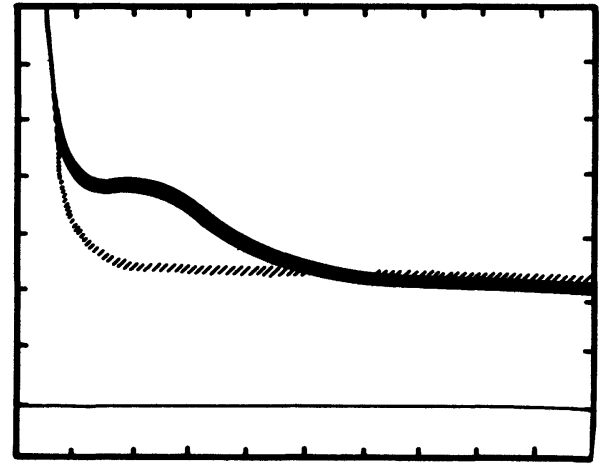

Fig. 1. - Time record of the current $I$ flowing through the sample. The continuous curve corresponds to the sample in the $\mathrm{C}$ phase $\left(T-T_{\mathrm{c}}=-1.5^{\circ} \mathrm{C}\right)$, the broken curve to the $\mathrm{A}$ phase $\left(T-T_{\mathrm{c}}=0.8^{\circ} \mathrm{C}\right)$. One division of time is $0.5 \mathrm{~ms}$. The inversion of the orienting electric field is just at the left of the image. One division of $I$ is $0.5 \mu \mathrm{A}$. Each curve is the superposition of ten records on the oscilloscope screen, taken at the frequency of $17 \mathrm{~Hz}$ with an electric field of $\pm 725 \mathrm{~V} / \mathrm{cm}$.

an exponential of time constant $0.19 \mathrm{~ms}$ corresponding to a sample capacity of about $200 \mathrm{pF}$ and a constant conduction term of about $1.1 \mu \mathrm{A}$ corresponding to a sample resistance of $26 \mathrm{M} \Omega$ and a resistivity of $3.2 \times 10^{8} \Omega \mathrm{cm}$. In the $\mathrm{C}$ phase for the same $E$, at $T-T_{\mathrm{c}}=-1.5^{\circ} \mathrm{C}$, one can observe a bump of current, superimposed on the same exponential decay and on a light lower conduction term. We identify this bump as the current flow induced by the orientation of the dipoles for the three following reasons : first, the sign of this current is correct (we observe an increase of current corresponding to the increase of capacitance due to the orientation of the dipoles); secondly, its time duration (1 ms) compares well with the known [2] relaxation frequency $(400 \mathrm{~Hz})$ in the absence of strong applied field; third, this time duration varies with the field but does not vary with its frequency, under $200 \mathrm{~Hz}$.

To obtain the value of the polarization we compute graphically the integral of the bump of current. Working at different values of the orienting field $3 E_{\mathrm{c}}<E<10 E_{\mathrm{c}}$ the bump duration varies from 1 to $2 \mathrm{~ms}$, but we have checked that its integral is constant and equal to 3.45 stat coulomb for this temperature.

Using this method, we have measured the ferroelectrical polarization at different temperatures. We have explored the range from $69.2^{\circ}$ to $65.9^{\circ}$, the beginning of the $\mathrm{C}$ phase on our particular sample where impurities lower considerably the $\mathrm{A}$ to $\mathrm{C}$ transition-temperature (normally around $78^{\circ} \mathrm{C}$ for a pure sample). Our results are plotted in figure 2 . The order of accuracy of our measurement is $30 \%$ as is shown by the two points at $68.9^{\circ}$ obtained at the beginning and at the end of the experiment. The variation of $T_{\mathrm{c}}$ with the time and our naive graphical method of recording and integrating lead to the poor precision. As suggested by R. B. Meyer [1] $P$ should be proportional to the tilt angle of the molecules in the layers $\left(P=P_{0}\right)$. The angle $\theta$ has been recently 


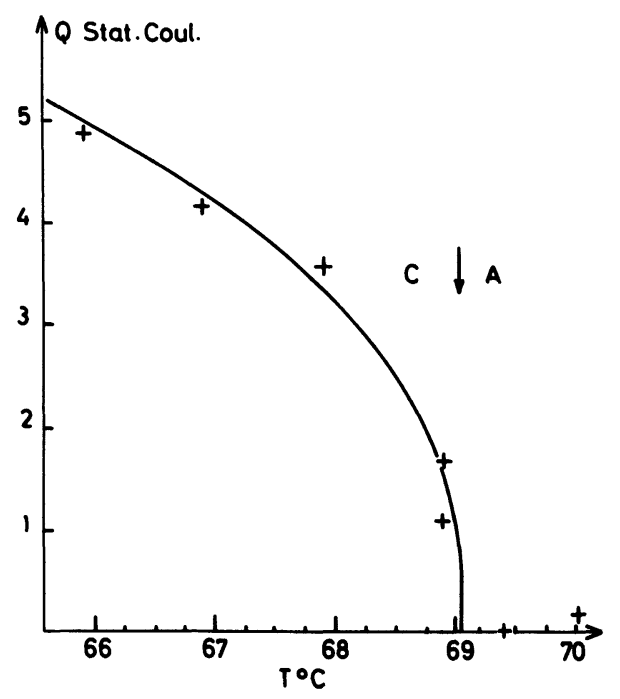

Fig. 2. - Plot of the electrical charge $Q$ induced by the reversing of the permanent polarization $P$ of the sample versus the temperature $(Q=2 P S$, where $S$ is the sample area perpendicular to the field). The continuous curve is the best fit to a law of the form $\left(T_{\mathrm{c}}-T\right)^{0.4}$. Two points at the same temperature close to $T_{\mathrm{c}}$ made at the beginning and at the end of the experiment give an estimate of the accuracy.

measured [6] and behaves as $\left(T_{\mathrm{c}}-T\right)^{0.4}$. We have drawn in figure 2 a fit of our data to a law of the form $\left(T_{\mathrm{c}}-T\right)^{0.4}$. As can be shown, the agreement is reasonable. From this fit we can derive $P_{0}=0.05$ Debye $/ \mathrm{mol}$.rad. for the permanent polarization of this compound versus its tilt angle.

On the same compound, the value of $\boldsymbol{P}_{0}$ has been evaluated through the measurement of the critical field necessary to unwind the helical texture [3] : the present value is three times larger. This is not too surprising; the twist elastic constant is not known and the screening effect from residual charges tends to lower the observed $P_{0}$ in the electroelastic experiment. In our experiments, these residual charges contribute to the conduction and may also to the capacitance. We do not think that the diffusion charges play any role in our experiments, first because with a typical diffusion coefficient $D=10^{-6} \mathrm{~cm}^{2} / \mathrm{s}$ we found a diffusion time of about $200 \mathrm{~s}$, much larger than the duration of the observed bump, and second because any spurious effect from these charges should also be visible in the A phase.

In conclusion, we think that, for the first time, we have measured the absolute polarization $P_{0}$ of a ferroelectric liquid crystal. Up to now our accuracy is not very good. This lack of accuracy prevents us presently from extending this measurement to the other compounds of lower polarization. We are building an improved set-up for that purpose. The obtained value of 0.05 Debye/mol.radian is to be compared to the transverse dipole $\mathfrak{T}$ of the molecule which comes mostly from the chlorine substitute on the chiral carbon and is of the order of two Debye. The smallness of the ratio $P_{0} / \mathfrak{S} \sim 10^{-2}$ means that, even for this strong ferroelectric smectic $\mathrm{C}$ phase, the molecules rotate almost freely around their long axis.

We are very indebted to Dr. Liebert and P. Keller for the synthesis of the compound used in this experiment.

\section{References}

[1] Meyer, R. B., Liebert, L., Strzelecki, L. and Keller, P., J. Physique Lett. 36 (1975) L-69.

[2] Duke, R., Durand, G., Martinot-Lagarde, Ph., to be published in the Proceedings of the Sixth International Liquid Crystal Conference, August 1976.

[3] Keller, P., Liebert, L., Strzelecki, L. and MartinotLAGarde, Ph., to be published in the Proceedings of the Sixth International Liquid Crystal Conference, August 1976.

[4] Pieranski, P., Guyon, E. and Keller, P., J. Physique 36 (1975) 1005.

[5] Yu, L. J., LeE, H., BaK, C. S. and Labes, M. M., Phys. Rev. Lett. 36 (1976) 388.

[6] Martinot-Lagarde, Ph., J. Physique Colloq. 37 (1976) C3-129. 